\title{
Gain Profile Optimization for Raman+EDFA Hybrid Amplifiers with Recycled Pumps for WDM Systems
}

\author{
Márcia M. Jardim Martini ${ }^{1,3}$; Carlos Eduardo S. Castellani²; Maria José Pontes²; Moisés R. N. \\ Ribeiro $^{2}$; and Hypolito José Kalinowski ${ }^{1}$ \\ 1-Federal University of Technology - Paraná, Av. Sete de Setembro 3165, 80230-901, Curitiba-PR, Brazil, \\ marciamjmartini@yahoo.com.br \\ 2-Federal University - Espírito Santo - Av. Fernando Ferrari 514 - Campus Goiabeiras, 29060-970, Vitória- \\ ES, Brazil \\ 3- Federal Center for Technology Education - Minas Gerais - Av. Amazonas 5352, Nova Suíça, Belo \\ Horizonte-MG, Brazil.
}

\begin{abstract}
An approximated technique to quickly optimize the gain profile of multi-pump broadband hybrid amplifiers under residual pump recycling in WDM systems is proposed. It is based on the amplifier spectral equalization by adjusting the flexible gain profile of a multi-pump Raman amplification stage to compensate the Erbium-doped stage gain variation within the $\mathrm{C}$-band. In order to increase the pump power efficiency, the Erbium-doped fiber section recycles the residual Raman pump. Three different cases considering, respectively, one, two, and three pumps are analyzed to demonstrate the effectiveness of the proposed technique. The characterization of the Raman+EDFA hybrid amplifier has been performed in terms of global gain, ripple, and noise figure. The proposed optimization method for the Raman pump sets allows ripple figures to be reduced by half of their original values in single pumped configurations. Our results also suggest that two pump lasers is the best configuration when the maximum gain, minimum ripple, and pump power efficiency are simultaneously considered.
\end{abstract}

Index Terms - EDFAs, hybrid optical amplifiers, pump recycling, stimulated Raman scattering, WDM.

\section{INTRODUCTION}

Dense wavelength division multiplexing (DWDM) is one of the most suited methods of increasing the transmission capacity of an optical fiber system. It offers an attractive solution to increase bandwidth without changing the already installed fiber cables [1]-[5]. Raman amplification is a promising technique to expand the reach in optical transmission systems. There are even methods to provide compensation for both fiber loss and dispersion [6], [7]. Raman amplifiers can also be used along with the high output power Erbium-Doped Fiber Amplifiers (EDFA). This hybrid amplifier configuration adds spectral shaping flexibility to develop broadband devices [8].

Diverse configurations have been proposed in the literature, from discrete modules of amplification to hybrid amplifiers using residual Raman pump recycling schemes. One of the main goals lies in the improvement of the global power conversion efficiency [9]-[16]. Previous work reported independent modules for Raman and EDF amplification, which have individual pumping scheme. The Raman 
amplification has been achieved either along the propagating fiber [9]-[10] or using shorter lengths of specific fibers within an amplifier module [12]-[16]. As far as the pump and signal propagation are concerned, there are both co- and counter-propagating arrangements [9]-[16]. More recently, the hybrid Raman+EDF amplifiers attracted attention in terms of more efficient use of the available pump energy [17]. This paper investigates such configuration with the aim of optimizing their systemic features, in particular their gain profile characteristics.

Lee et al. [12]-[14] have experimentally compared the performance of hybrid Raman+EDFA amplifiers, in which the Raman gain is provided in a dispersion compensating fiber (DCF) section of fiber. In their works the residual Raman pump power is recycled to pump the EDFA stage. Several different configurations based on a single pump laser over DCFs were studied. They found that by recycling the residual Raman pump in a cascaded erbium-doped fiber (EDF) section, as a secondary signal amplification stage, pump conversion efficiency can be increased. The amplifier characterization has been calculated in terms of global gain, and noise figure (NF). However, it has been restricted to the single channel characterization only, disregarding the ripple parameter within the amplifier effective gain bandwidth, which is a very important issue in WDM optical communication systems.

Tiwari et al [15] have performed experimental single and multi-channel investigations, where the signal first enters the Raman stage before undergoing further amplification in cascaded EDF section. More recently, numerical simulation has been employed in order to add flexibility to the analysis and design of such hybrid amplifiers [16]. Simulation has become an important tool for finding out the best configuration before the experimental implementation phase. Nevertheless, the time-consuming numerical methods in these simulations are an issue yet to be properly addressed. A multi-pump Raman amplifier optimization is an intricate multi-objective problem that needs to be carefully analyzed.

In this paper, the authors take advantage of multi-pump spectral shaping capabilities of the Raman section in the hybrid amplifier in order to equalize the EDFA's gain profile section increasing the global amplifier bandwidth. The main contribution of this paper is the proposal of an optimization procedure for the multi-pumped Raman section, regarding its wavelengths and pump powers, to play the role of global gain equalizer of the hybrid amplifier. Moreover, this optimized hybrid configuration may also improve amplifier characteristics towards the forthcoming WDM systems with efficient use of energy by recycling the residual Raman pump to EDF section instead of using separate pumping system.

The main challenge for the design of such hybrid devices lies in setting up the wavelengths and powers for the Raman pumping lasers. Moreover, these parameters will directly influence both the amount and the efficiency of the residual pump power that will be left for the EDF section to improve the global amplifier performance. Raman amplification has to be modeled by nonlinear coupled equations concerning the pump-pump and pump-signal interactions. There is no simple relation 
between gain, wavelength, and power of the multiple pump lasers that can be found without seriously compromising the accuracy of the numerical solutions. As a result of these intricate problems, the current proposals in the literature are mostly limited to arbitrary (i.e., non-optimized) parameters for the hybrid amplifiers setup [12]-[16].

A new optimization technique to design flatter gain hybrid amplifiers to use in WDM systems is here proposed. Our technique is inspired in the concept of geometric compensating scheme proposed by $\mathrm{Li}$ et al. [18] for Raman amplifiers. The optimization has been carried out on a hybrid Raman+EDFA amplifier configuration, using DCF with multiple pump lasers on the Raman amplification stage. The proposed multi-pump optimization has been focused on the global gain and its ripple factor for the hybrid amplifier. The latter aspect has been largely neglected in previous investigations dealing with such amplifiers, e.g., [12] - [16], but flat gain, i.e., low ripple, is a major requirement in present-day WDM systems. The method discussed in this work extends the spectral analyses reported in [17] to the study case of WDM input channels. It has been shown that the multichannel gain spectrum of the hybrid amplifier is quite different from the profile observed with a single channel spectral analysis. Furthermore, the gain ripple is affected by the signal input power [15], [16].

An analytical approximated model [19] is used for the spectral shaping of the Raman gain stage, avoiding the time-consuming process of simulation-based spectral profile optimization by searching for the best set of pumping parameters. Thus, the numerical simulation procedure is only employed in order to obtain the global performance of specific Raman+EDFA setup configurations.

The remainder of this paper is organized as follows. Section II describes the proposed optimization methodology. Section III brings the results for two and three-pumped hybrid amplifiers. Finally, the conclusions are drawn in Section IV.

\section{PROPOSED METHODOLOGY}

The optimization of the pump wavelengths and powers is a critical task that has to be performed when designing a Raman amplifier. Most of the attempts to accomplish this do not provide good estimates for Raman amplifier characteristics without using numerical methods at high computational cost. Nevertheless, an accurate approximated analytical method that enables the calculation of signal gain profile has been proposed by Cani et al. [19]. Based on this method, we put forward an optimization technique to design broadband hybrid amplifiers by adjusting the Raman amplifier parameters that enables flatter and higher global gain profiles. The method is composed of two phases. The first one searches for a set of "good solutions" in a large search space. In order to reduce the computational cost, a fixed EDF gain profile is considered along with the above mentioned approximated analytical solution for the Raman section. In the second phase, however, a precise numerical representation for all components in the hybrid amplifier is employed to identify the best one among the set obtained from the first phase. In other words, a coarse search based on an analytical method helps to identify potential solutions to the problem of hybrid amplifier design. 
Then, the refined (and time-consuming) numerical simulation is used to pin down the best solution out of the set of potential solutions. These phases are implemented as follows:

\section{A. First Phase}

A large population of pumping setup with 30,000 different configurations is generated by randomly selecting (under uniform distribution) wavelengths and pump powers across a given practical range for hybrid amplifiers. Provided that residual Raman pump is recycled in the EDF stage, a wavelength range was set as 1400-1480 nm. A typical commercially available Raman pump lasers power range (50-300 mW) was considered. The analytical method proposed by Cani et al [19] allows the evaluation of Raman gain profile of this large set of pump configurations, with very reasonable accuracy, within one minute of computation time using Matlab 7.0 running on Intel ${ }^{\circledR}$ Core $^{\mathrm{TM}} 2$ Duo Processor@2.66 GHz with 2 GB RAM.

Differently from optimizations performed in [19] that solely deal with a Raman stage the aim here is not only to find the best Raman gain but also to match it to the EDF gain profile to obtain higher and flatter global gains. A typical EDF gain profile is obtained under different pumping conditions and it is geometrically approximated by two linear segments, independently of the actual residual pumping and input signal power as described in [17]. Therefore, at this phase, neither EDF nor Raman gain saturation are taken into account.

The pump set (wavelengths and powers combinations) that produces adequate global gain profile, i.e., the Raman gain that summed to the approximated EDF gain stage results in flatter gain, is then selected. The pumping setups meeting the criteria described above are then qualified for the second round of the selection process. For the studied case, it is usually limited to 10 configurations.

\section{B. Second Phase}

The second round of setup selection is manually performed using the OptiSystem ${ }^{\circledR}$ 7.0, in which the whole hybrid amplifier is now included in the calculations through accurate numerical methods. The use of this commercial simulator enables us to take into account effects such as amplified spontaneous emission (ASE) and double Rayleigh backscattering (DRS) in the analysis of the EDFA+Raman hybrid amplifier. The EDFA full numerical model in OptiSystem 7.0 with parameters taken from [12] (and the default ones otherwise) has been used. This enables us to consider effects such as amplified spontaneous emission, excited state absorption, Rayleigh scattering background loss wavelength and power dependence. Er-Er ion interaction effects, ion-ion interaction effects and temperature dependence are also considered.

The Raman amplifier numerical (Average Power) model of the used OptiSystem 7.0 software includes pump-to-pump, signal-to-signal and pump-to-signal Raman interactions, spontaneous Raman emission and its temperature dependency, stimulated Raman scattering, pump depletion due to Raman energy transfer, high-order Stokes generation, multiple Rayleigh backscattering, fiber loss and 
spontaneous emission noise. Once again, default parameters have been used when there were not given by [12].

Although the pump depletion by signal and the actual residual pump to the EDF are not considered in the First Phase, the comprehensive numerical methods used in OptiSystem ${ }^{\circledR} 7.0$ will allow us to rule out solutions affected by such neglected features. The smallest ripple obtained for the hybrid Raman+EDFA amplifier found among the 10 selected configurations defines the final pump setup parameters.

Multiple channels gain is carried out using eight signal wavelengths given by $\lambda_{1}$ to $\lambda_{8}$ starting from $1535 \mathrm{~nm}$ and spaced by $0.5 \mathrm{~nm}$. The total signal input power levels of $-20 \mathrm{dBm},-10 \mathrm{dBm}$, and -5 $\mathrm{dBm}$ are used. This means, respectively, $-29 \mathrm{dBm},-19 \mathrm{dBm}$, and $-14 \mathrm{dBm}$ per channel.

The configuration based on Lee et al. [12], presented in Figure 1, has been used for the analysis in this paper. A Raman amplifier using a $12.6 \mathrm{~km}$ DCF with effective area of $15.3 \mu^{2}$ with attenuation coefficient of $0.5 \mathrm{~dB} / \mathrm{km}$, at $1550 \mathrm{~nm}$, dispersion coefficient of $-98 \mathrm{ps} /(\mathrm{nm} . \mathrm{km})$ composes the first stage. The second stage is given by an EDFA using a $10 \mathrm{~m}$ of EDF section (Er doping radius $=2.2$ $\mu \mathrm{m}$, core area $=15.2 \mu \mathrm{m}^{2}$ and Er concentration $=1 \times 10^{25} \mathrm{~m}^{-3}$ ). The Giles parameters (cross-section) considered in the simulations are the default values available in the OptiSystem 7.0 to the erbium doped fiber, since no information regarding the EDF were available in the mentioned references. The optimization is performed for amplifiers with two and three pump lasers. The optical isolators, WDM couplers, pump combiners, and splicing and fiber connectors are herein assumed as ideal components. We have considered the counter-propagating configuration due to its better power conversion efficiency [1]-[4]. In addition, it has checked through simulations in OptiSystem® 7.0 that the noise figure for the hybrid amplifier (under the three different input power levels mentioned above) is, approximately, $1.5 \mathrm{~dB}$ lower than the co-propagating configuration. It is important to highlight that the average gain and ripple values are nearly the same in both configurations.

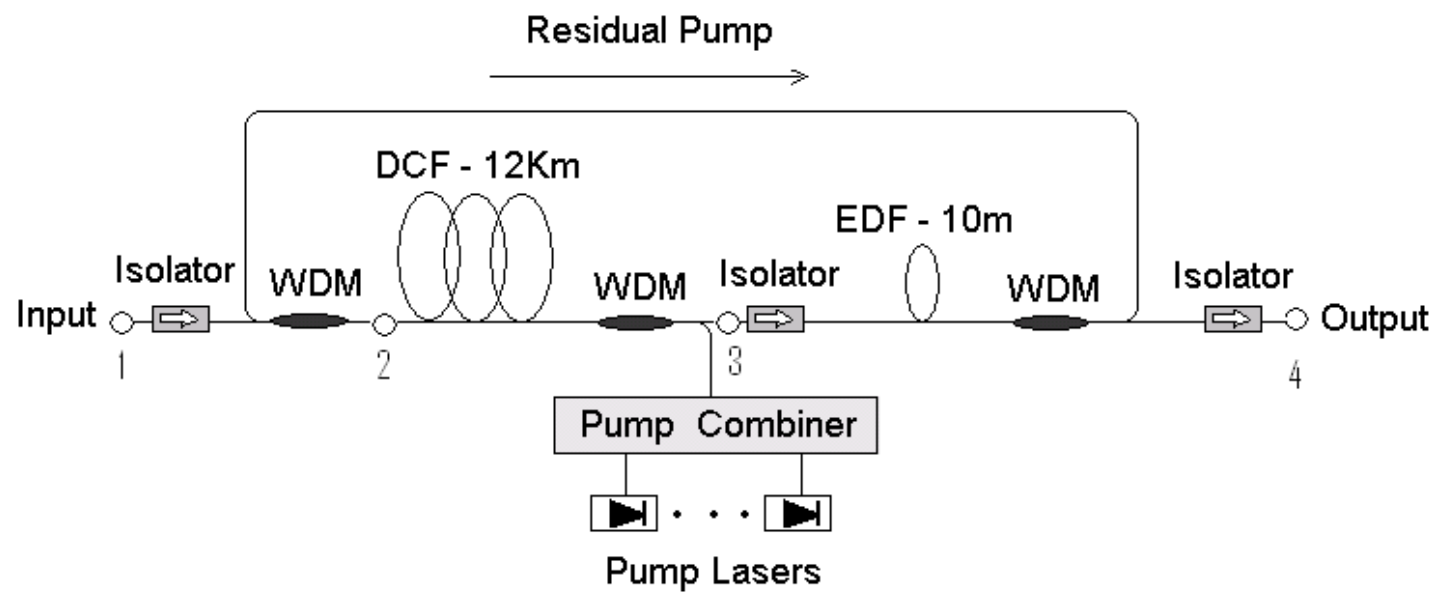

Figure 1: Schematic design of the hybrid amplifier (by Lee et al. [12]) under counter-propagating configurations for both Raman and EDF amplifying stages. The monitoring points for gain assessment are numbered from 1 to 4 . 
The average global gain $\left(G_{\text {average }}\right.$ ) has been evaluated considering the global gain for each channel $\left(\mathrm{G}_{\mathrm{ch}}\right)$ divided by the number of channels $\left(\mathrm{N}_{\mathrm{ch}}\right)$, as given by the expression (1),

$$
\mathrm{G}_{\text {average }}=\frac{\sum \mathrm{G}_{\mathrm{ch}}}{\mathrm{N}_{\mathrm{ch}}}
$$

The calculated Raman gain $\left(G_{\text {Raman }}\right)$, according to the layout shown in Fig. 1, corresponds to the ratio between the signal power at point 3 and the input power at point 2 named as first stage. The expression is,

$$
G_{\text {Raman }}=\frac{P_{3}}{P_{2}}
$$

It means $P_{3}$ gives the output power at point 3 and $P_{2}$ is the input power at point 2 .

On the other hand, the EDFA gain $\left(G_{E D F A}\right)$ is determined through the ratio between the output and the input powers, respectively, at point 4 and point 3 . This fiber section is named as second stage. The expression is,

$$
G_{E D F A}=\frac{P_{4}}{P_{3}}
$$

In this case, $P_{4}$ is the output power at point 4 and $P_{3}$ gives the input power at point 3.

Similarly, the global gain of the hybrid Raman+EDFA $\left(G_{\text {global }}\right)$ is given by the ratio of the output power at point 4 and the input power at point 1, resulting in the expression (4)

$$
G_{\text {global }}=\frac{P_{4}}{P_{1}}
$$

The ripple value corresponds to the difference between the highest $\left(G_{c h}^{\max }\right)$ and the lowest $\left(G_{c h}^{\min }\right)$ global gain values, which covers approximately the whole C-band. It is given by:

$$
\text { Ripple }=G_{c h}^{\max }-G_{c h}^{\min }
$$

\section{RESULTS}

We have analyzed three different cases regarding the number of pump lasers (single pump, two, and three pumps). Global gain and ripple results indicate the effectiveness of the proposed technique to EDFA gain profile equalization using the pump optimization of the Raman amplifier for a WDM environment using 8 channels simultaneously coupled to the input fiber. The gain profile to each amplifying stage, as well as the resulting optimized hybrid amplifier with multi-pumping, has been analyzed considering different signal input power per channel. 
In order to illustrate the potential of the proposed method, a single pumped hybrid amplifier fed with $500 \mathrm{~mW}$ is presented for comparisons purposes. This is a usual power level found for pumping in the literature for recycled Raman+EDFA hybrid amplifiers, e.g., [12] - [16].

The pump wavelength has been set at $1425 \mathrm{~nm}$ so that the resulting Raman gain would be around $1535 \mathrm{~nm}$. Figure 2 brings the outcomes for $G_{\text {Raman }}, G_{E D F A}$, and $G_{\text {global }}$, calculated according to equations (2) - (4) under total signal input power set at $-10 \mathrm{dBm}$. The results in Fig. 2 indicate the channels at shorter wavelengths are mainly amplified by the Raman section, whereas the channels at longer wavelength mostly receive gain from the EDF section, as Raman amplification approaches 0 $\mathrm{dB}$ for $1555 \mathrm{~nm}$ and longer wavelengths. Bear in mind that the EDF typical gain peak at $1530 \mathrm{~nm}$ is not present as in Fig. 2 since the input channels start at $1535 \mathrm{~nm}$. For the proposed hybrid configuration, the average global gain values were around $30.4 \mathrm{~dB}, 25.9 \mathrm{~dB}$, and $22.8 \mathrm{~dB}$ under -20 $\mathrm{dBm},-10 \mathrm{dBm}$, and $-5 \mathrm{dBm}$ of total signal input power, respectively. And the corresponding ripple values are $18.7 \mathrm{~dB}, 15.6 \mathrm{~dB}$, and $13.2 \mathrm{~dB}$. The average noise figure is $9.9 \mathrm{~dB}$, and it is virtually insensitive to the adopted values of signal input power. Similar results are found in experimental investigations using the setup shown in Fig. 1 [12] - [14]. The lower ripple values for higher signal input power is mainly due to EDF gain saturation while the Raman gain curve remains unaffected in this range of signal input power. The ripple reduction in this non-optimized result can be explained by the fact that the further EDFA saturation seems, by chance, to improve the symmetry between the curves producing, therefore, a flatter (global) hybrid gain. When our optimization method is applied, this kind of curve mirroring between Raman and EDFA is intentionally sought.

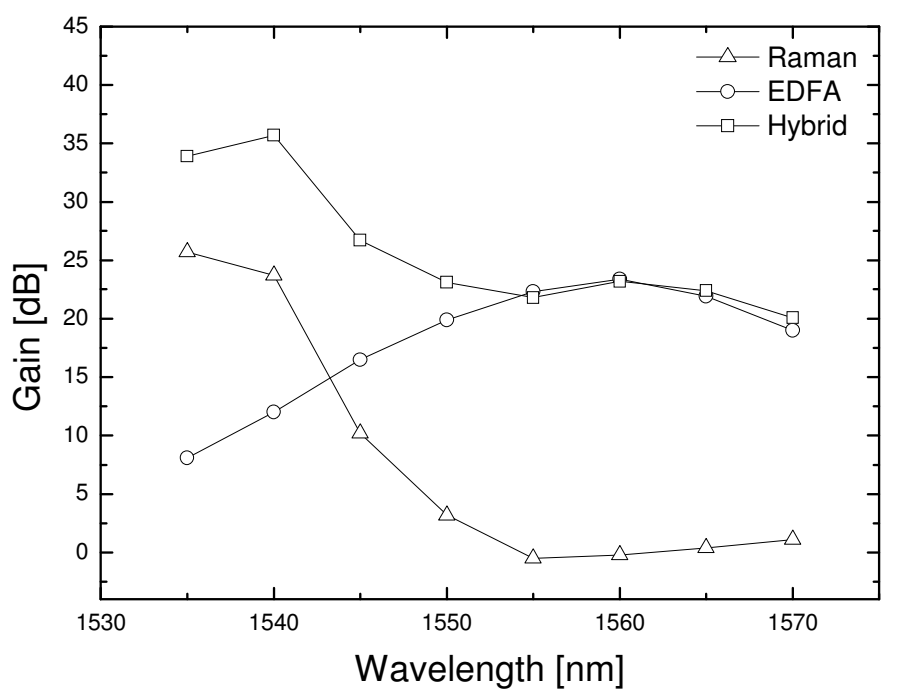

Figure 2: Gain profile considering one counter-propagating Raman pump at $1425 \mathrm{~nm}$ with $500 \mathrm{~mW}$ and signal input power of $-10 \mathrm{dBm}$.

Although the average gain for this particular setup meets commercial in-line amplifier requirements, its ripple levels are unacceptable for practical WDM systems (e.g. [20]). Our solution 
to this problem is found by reshaping the gain profile of the Raman stage. This includes not only another pump laser, but also a careful optimization of their wavelengths as well as their pump levels. The best outcome, according to our method described in Section II, for using two pump lasers in the setup in Fig. 1 is $1425.0 \mathrm{~nm}$ and $1468.4 \mathrm{~nm}$ with powers of $296.3 \mathrm{~mW}$ and $61.3 \mathrm{~mW}$, respectively.

Figure 3 shows the Raman, EDFA and global gain spectral profiles for this optimized hybrid amplifier, considering signal input power equal to $-10 \mathrm{dBm}$. In comparison to the single pumped configuration with this input signal level, the average global gain is improved from $25.9 \mathrm{~dB}$ to 28.8 $\mathrm{dB}$ while the difference between signal output powers is reduced more than tenfold (as ripple improves from $15.6 \mathrm{~dB}$ to $4.3 \mathrm{~dB}$ ). It can be seen in Fig. 3 that the Raman gain profile nearly mirrored the EDF section counterpart. As a result, the total gain profile becomes much flatter than the previous case, in which only one pump was used and no gain profile optimization was formally considered. For different total signal input power of $-20 \mathrm{dBm}$ and $-5 \mathrm{dBm}$, the average global gain are $34.3 \mathrm{~dB}$ and $24.3 \mathrm{~dB}$ with ripple figures of $7.0 \mathrm{~dB}$ and $6.5 \mathrm{~dB}$, respectively.

In contrast with the single-pumped amplifier in Fig 2, the Raman stage amplifies even channels at longer wavelengths. The recycling of the remaining pump power in the EDF section results in an almost flat spectral gain in Fig.3. Investigation for different levels of input signal powers is presented in Fig. 4 (only for the EDFA gain profile since the Raman stage remains unchanged). It is noteworthy the effect of gain saturation taking place in the EDF section, this probably happens due to the EDF to be placed just after the Raman module and receive higher signal levels. Moreover, notice that the EDF stage is pumped by the amount of pump left over by the Raman amplifier. As the signal level is increased, the pump is more depleted by the input signal in the Raman stage and, as a result, there is less pump power left to the EDF module.

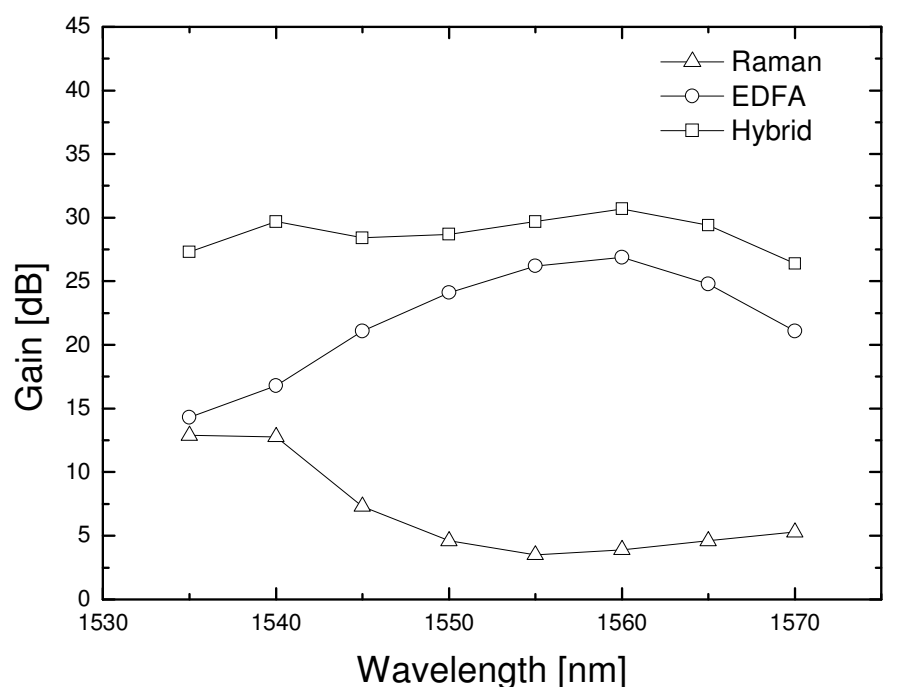

Figure 3: Gain profile for the optimized hybrid amplifiers with 2 pumps: $1425.0 \mathrm{~nm}$ and $1468.4 \mathrm{~nm}$ with powers of 296.3 $\mathrm{mW}$ and $61.3 \mathrm{~mW}$, respectively. $\mathrm{P}_{\mathrm{s}}=-10 \mathrm{dBm}$. 


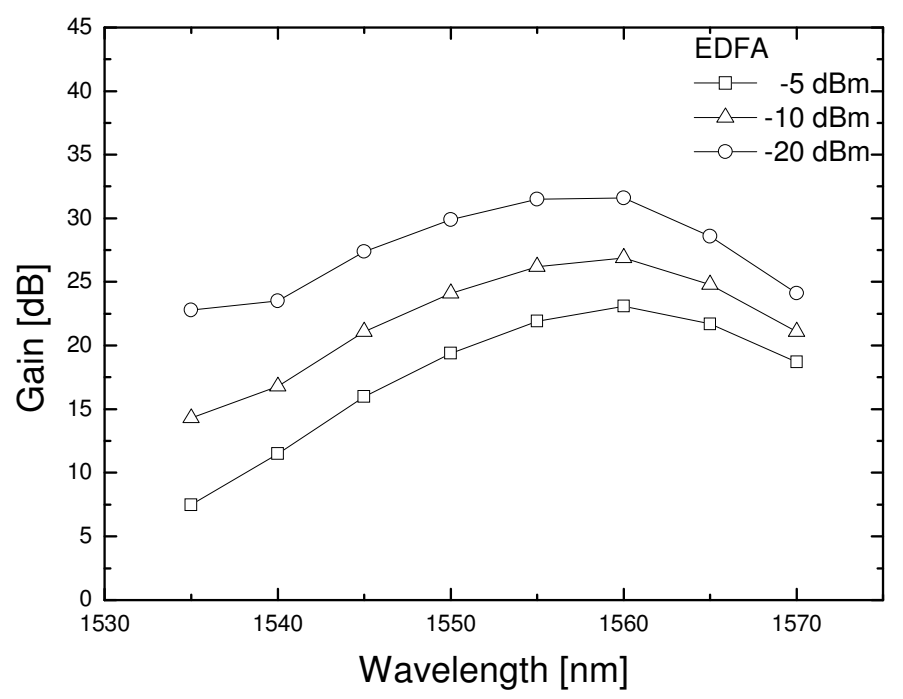

Figure 4: EDFA gain profile saturation analysis for the optimized hybrid amplifiers with 2 pumps of $1425.0 \mathrm{~nm}$ and $1468.4 \mathrm{~nm}$ with powers of $296.3 \mathrm{~mW}$ and $61.3 \mathrm{~mW}$, respectively.

Figure 5 shows the NF profile of the optimized hybrid amplifier, using the conditions that have been considered to obtain the results shown in Fig. 3 and Fig. 4. Noise figure has a slight increase in the region from $1550 \mathrm{~nm}$ to $1560 \mathrm{~nm}$ and NF seems to be insensitive to the signal input power level. It is important to highlight that NF also benefits from multi-pump schemes as its average value $(9.4 \mathrm{~dB})$ is reduced when compared with single-pumped case $(9.9 \mathrm{~dB})$.

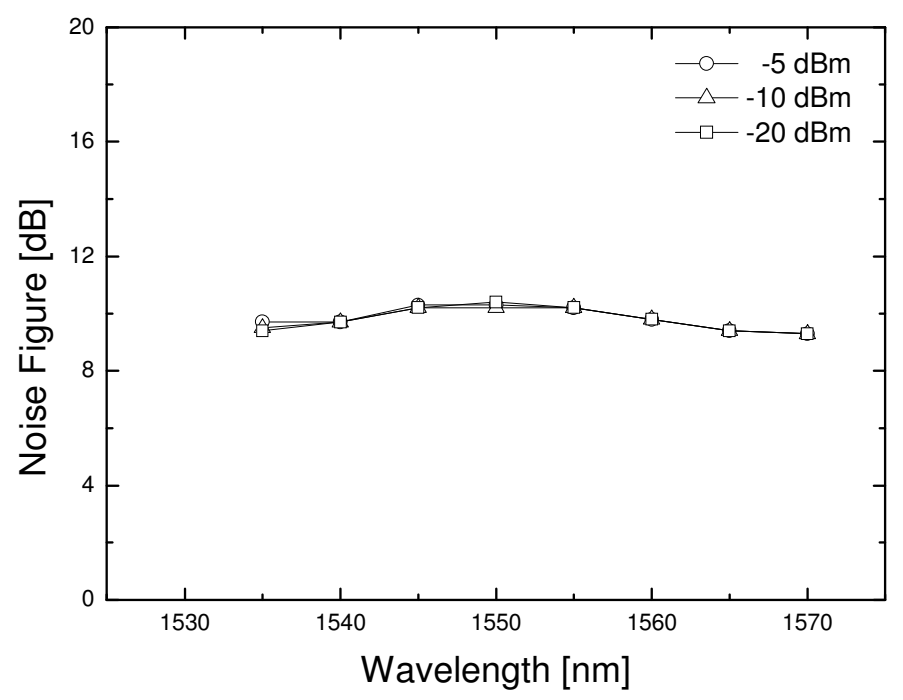

Figure 5: Noise figure against wavelength for the optimized hybrid amplifiers with 2 pumps and Ps $=-20 \mathrm{dBm},-10 \mathrm{dBm}$, and $-5 \mathrm{dBm}$.

In order to enhance even more the global gain and ripple profiles, a third Raman pump laser has been added to the pump module in Fig. 1. Applying our method to this new scheme, the pumps that enabled the best gain profile to the hybrid amplifier are $1414.5 \mathrm{~nm}, 1426.5 \mathrm{~nm}, 1472.6 \mathrm{~nm}$ with pump powers of $244.1 \mathrm{~mW}, 269.9 \mathrm{~mW}$, and $60.1 \mathrm{~mW}$, respectively. It is shown in Fig. 6 the gain profiles of 
the hybrid amplifier, Raman amplifier and the EDFA for simulations considering three pump lasers and $-10 \mathrm{dBm}$ for the signal input power. The Raman stage has now higher amplification levels than the two-pump scheme, with higher gain for the longer wavelengths, which implies an overall higher gain for all input channels. When it is combined to the EDFA amplification, the hybrid amplifier presents $G_{\text {global }}$ of $31.2 \mathrm{~dB}$.

However, it is noteworthy the fact that the EDFA peak gain at $1560 \mathrm{~nm}$ is $3.2 \mathrm{~dB}$ below the value obtained for two-pump scheme. The average global gain values for $-20 \mathrm{dBm}$ and $-5 \mathrm{dBm}$ of signal input power are $37.7 \mathrm{~dB}$ and $26.2 \mathrm{~dB}$, respectively. It confirms the previous observation that the EDFA is operating under saturation due to the higher output signal levels of Raman stage, despite the fact that there is more remaining residual pump available to the EDF section.

Contrary to the expectations, the ripple values found for the signal input power levels of $-20 \mathrm{dBm},-10 \mathrm{dBm}$, and $-5 \mathrm{dBm}$ are, respectively, $5.4 \mathrm{~dB}, 9.3 \mathrm{~dB}$, and $12.6 \mathrm{~dB}$. Therefore, only in the small signal regime there is benefits from a third pump, while the saturation EDF effect makes the ripple figures worst than in the two-pump scheme. Although the bandwidth can be extended and the ripple reduced with more pump lasers in conventional Raman amplifiers [19], the intricate relationship between gain factors in the hybrid Raman+EDFA with recycling residual pump poses a different challenge when optimizing such amplifiers.

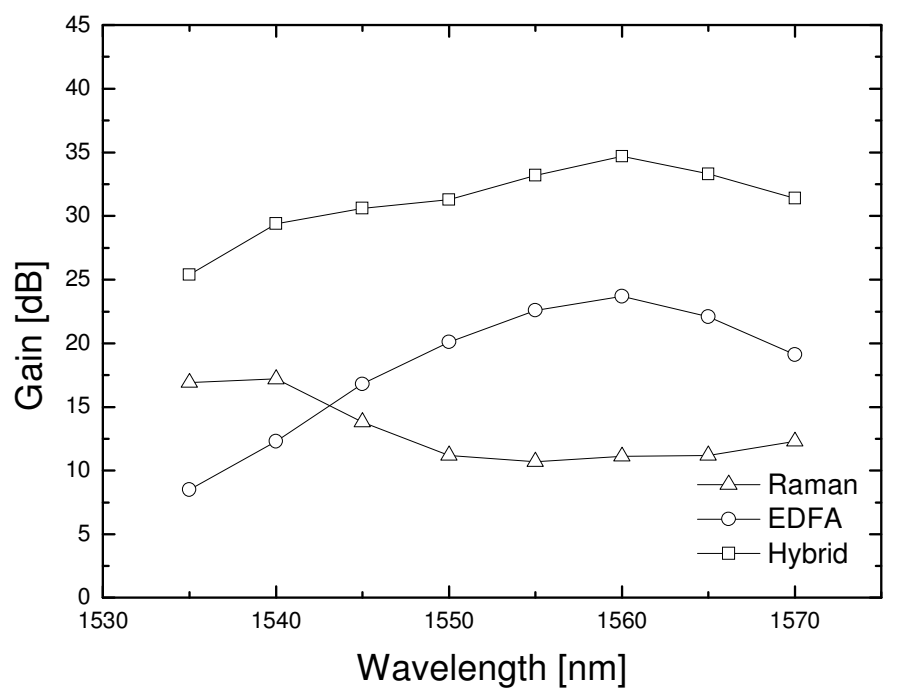

Figure 6: Gain spectrum for the optimized hybrid amplifiers considering with three pumps of $1414.5 \mathrm{~nm}, 1426.5 \mathrm{~nm}$, and $1472.6 \mathrm{~nm}$ with powers of $244.1 \mathrm{~mW}, 269.9 \mathrm{~mW}$, and $60.1 \mathrm{~mW}$, respectively.

Fig. 7 shows the NF as a function of signal wavelength with average value of $9.1 \mathrm{~dB}$ for the optimized hybrid amplifier with three pumps, which is close to the result with two pumps presented in Fig. 6. 


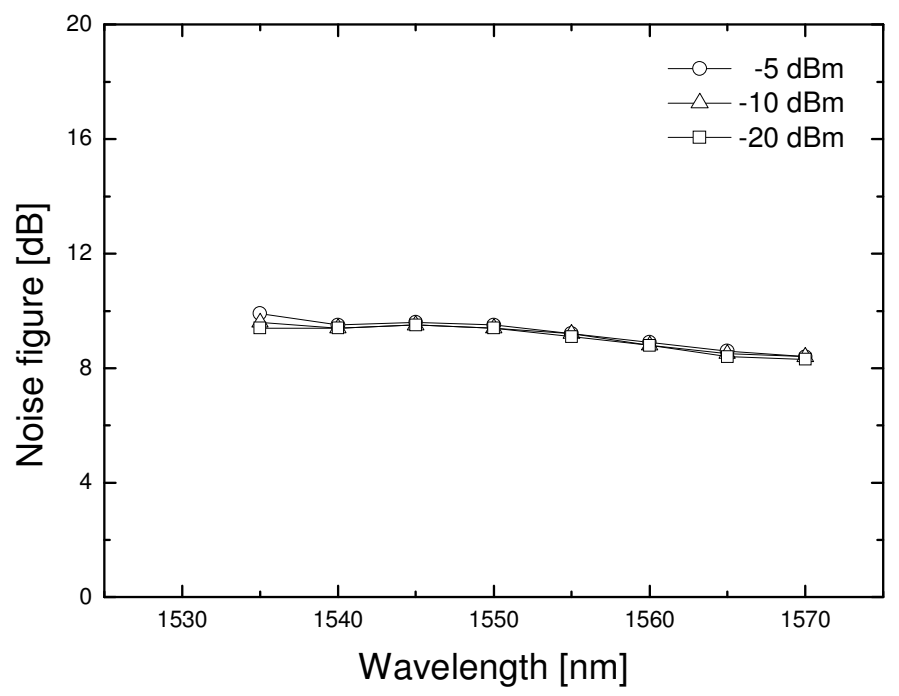

Figure 7: Noise figure against wavelength for the optimized hybrid amplifiers with 3 pumps and total signal input power of $-20 \mathrm{dBm},-10 \mathrm{dBm}$, and $-5 \mathrm{dBm}$.

The use of two or three pump lasers, instead of just one, enhances the global gain flatness of the EDFA+Raman hybrid amplifier as observed in separate Raman amplifiers [5]. In addition, the multipump approach here proposed can also be cost efficient since commercial low power Raman pump lasers (i.e., $<300 \mathrm{~mW}$ ) can be used instead of an expensive single high power pump laser (e.g., 500 $\mathrm{mW}$ ) that are usually employed in the literature [12] - [14]. Figure 8 summarizes the hybrid amplifier performance with one, two and three optimized pumping lasers. There is a general trend for ripple reduction and global gain improvement as the number of (optimized) pump lasers is increased.

It is important to highlight that the average gain of the Raman stage mostly benefits from higher total pump powers, but its spectral gain profile is very sensitive to the correct pump set selection and the EDF section operation regime. For instance, there is a slight increase in the ripple outcome in Fig. 8 when a third pump is added (under signal higher total input power, namely, $-5 \mathrm{dBm}$, and $-10 \mathrm{dBm})$. The saturation of the EDF section can be accounted for this result. The EDFA gain profile in the First Phase of our method does not consider its saturation effects, so that the Raman stage spectral profile does not equalizes its EDF counterpart as efficiently as it does in the non-saturated condition. Therefore, there is still room for improvements in (re)optimization methods aimed at further ripple reduction following the present proposals. Nonetheless, complexity may take its toll on processing time as other effects are included in the modeling. 


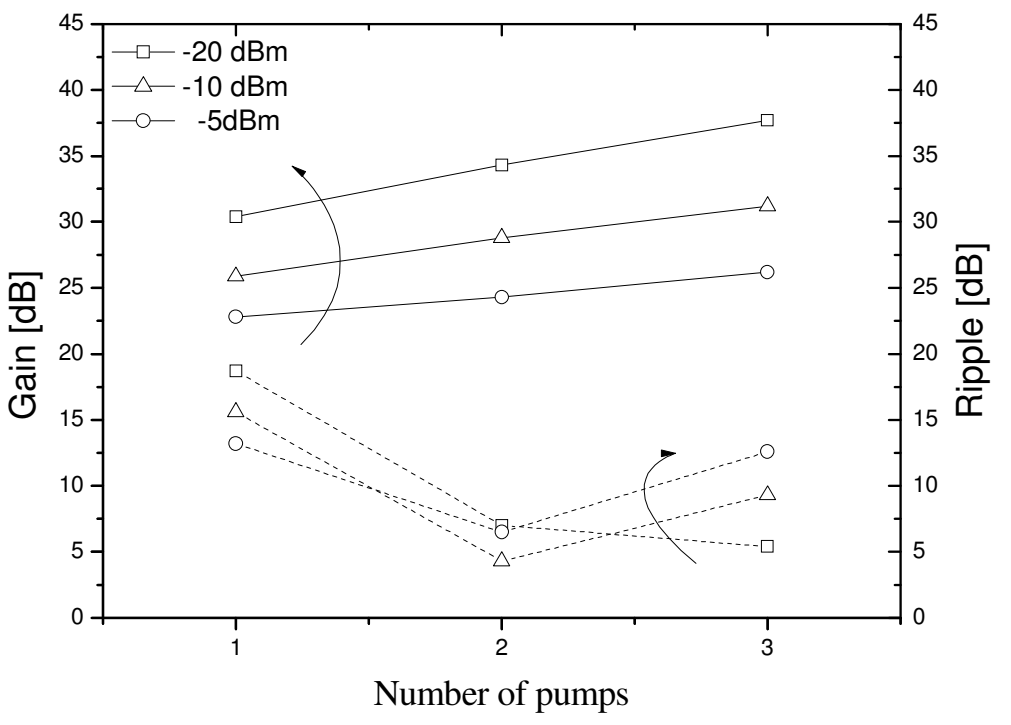

Figure 8: Ripple and average gain considering 1, 2 and 3 Raman pump lasers for three different total signal input power (-20 $\mathrm{dBm},-10 \mathrm{dBm}$ and $-5 \mathrm{dBm})$.

\section{CONCLUSIONS}

This paper proposed a method for the optimization of a multi-pumped EDFA+Raman hybrid amplifier configuration in which the recycling of the residual Raman pump is utilized in WDM systems. This optimization was made by adjusting the Raman gain profile to mirror the EDFA gain spectrum in order to get the minimal ripple values. The hybrid amplifier performance was evaluated in terms of global gain, ripple, and noise figure considering WDM input signals.

Simulations with one, two, and three pump lasers were performed searching for a flat global gain. For the investigated scenario under WDM input signals, the cost effective solution seems to be a two pump configuration as it improves the average gain and significantly reduces ripple figures while minor improvements were brought by adding a third pump. Moreover, all the cases presented similar noise figure with slight improvements when using the multi-pump scheme.

Our results demonstrated that this kind of hybrid amplifier, with the properly chosen pump wavelengths and powers, enables the design of broadband devices with enhanced power conversion efficiency. Optimal EDF length studies, considering specific input signal power levels, could lead to even better results as the EDFA gain saturation condition could be avoided.

\section{ACKNOWLEDGEMENTS}

The authors are grateful to the Federal University of Espírito Santo and Federal University of Paraná that enabled developing this work. Special thanks to the Federal Centre of Technological Education of Minas Gerais and CAPES, for the opportunity to make this work possible.

\section{REFERENCES}

[1] G. Agrawal, Fiber-Optic Communication Systems, 3rd ed., John Wiley \& Sons Inc., USA, 2002.

[2] C. Headley, and G. Agrawal, Raman Amplification in Fiber Optical Communication Systems, Elsevier Academic Press, USA, 2005

[3] G. Agrawal, Nonlinear Fiber Optics, 3rd ed., Academic Press, USA, 2001.

[4] P. Becker,Erbium Doped Fiber Amplifiers - Fundamentals and Technology, Academic Press, EUA, 1998. 
[5] M. Karásek, M. Menif, and A. Bellemare, "Design of Wideband Hybrid Amplifiers for Local Area Networks, IEEE Proc. Optoelectronic," vol. 148, no. 3, pp. 150 - 155, June 2001.

[6] P. B. Hansen, G. Jacobovitz-Veselka, L. Gruner-Nielsen, and A. J. Stentz, "Raman amplification for loss compensation in dispersion compensating fiber modules," Electronic Letters, vol. 34, no. 11, pp. 1136 - 1137, May 1998.

[7] C. E. S. Castellani, S. P. N. Cani, M. E. V. Segatto, M. J. Pontes, and M. A. Romero, "Design Methodology for Multipumped Discrete Raman Amplifiers: Case-Study Employing Photonic Crystal Fibers," Optics Express, vol. 17, no. 16, pp. $14121-14131,2009$.

[8] E. Desuvire, Erbium-Doped-Fiber Amplifiers: Principles and Applications, John Wiley \& Sons Inc., New York, 1994.

[9] H. Masuda, S. Kawai, K. Aida, "Ultra-wideband Hybrid Amplifier Comprising Distributed Raman Amplifier and Erbium-Doped Fibre Amplifier," Electronics Letters, vol. 34, no. 13, pp. 1342 - 1344, June 1998.

[10] A. Ahmad, M. A. Mas Izyani, A. K. Zamzuri, F. R. M. Adikan, S-J. Sheih, M. A. Mahdi, Experimental Validation of OSNR Enhancement Utilizing Hybrid Raman/Erbium Fiber Amplifiers, Microwave and optical Technology Letters, vol. 45, no. 4, pp. 333 - 335, May 2005.

[11] Y. Emori, K. Tanaka, S. Namiki, "100 nm Bandwdth Flat-Gain Raman Amplifiers Pumped and Gain-Equalized by 12 Wavelength - Channel WDM Laser Diode Unit," Electronics Letters, vol. 35, no. 16, August 1999.

[12] J. H. Lee, Y. M. Chang, Y. G. Han, S. H. Kim, H. Chung, and S. B. Lee, "Dispersion-Compensating Raman/EDFA Hybrid Amplifier Recycling Residual Raman Pump for Efficiency Enhancement," IEEE Photonics Technology Letters, vol. 17, no. 1, pp. 43 - 45, January, 2005.

[13] J. H. Lee, Y. M. Chang, Y. G. Han, H. Kim, H. Chung, and S. B. Lee, "Performance Comparison of Various Configurations of Single-Pump Dispersion-Compensating Raman/EDFA Hybrid Amplifiers," IEEE Photonics Technology Letters, vol. 17, no. 4, pp. 765 - 767, April, 2005.

[14] J. H. Lee, Y. M. Chang, Y. G. Han, H. Chung, H, Kim, and S. B. Lee, "A Detailed Experimental Study on SinglePump Raman/EDFA Hybrid Amplifiers: Static, Dynamic, and System Performance Comparison," IEEE Journal of Lightwave Technology, vol. 23, no.11, pp. 3484 - 3493, November 2005.

[15] U. Tiwari, K. Rajan, and K. Thyagarajan, "Multichannel Gain and Noise Figure Evaluation of Raman/EDFA Hybrid Amplifiers," Optics Communications, no. 281, pp. 1593 - 1597, 2008.

[16] U. Tiwari, K. Thyagarajan, and M. R. Shenoy, "Simulation and Experimental Characterization of Raman/EDFA Hybrid Amplifier with Enhanced Performance," Optics Communications, no. 282, pp. 1563 - 1566, 2009.

[17] M. M. J. Martini; C. E. S. Castellani; M. J. Pontes; M. R. N. Ribeiro; and H. J. Kalinowski, "Multi-pump Optimization for Raman+EDFA Hybrid Amplifiers under Pump Residual Recycling," International Microwave Optoelectronics Conference, pp. 117 - 121, Belém, Brazil, 2009.

[18] Z. Li., C. Lu, J. Chen, and C. Zhao, "Raman Amplifier Design Using Geometry Compensation Technique," Optics Express, vol. 12, no. 3, pp. 436 - 441, February, 2004.

[19] S. P. N. Cani, L. C. Calmon, M. J. Pontes, M. R. N. Ribeiro, M. E. V. Segatto and A. V. T. Cartaxo, "An Analytical Approximated Solution for the Gain of Broadband Raman Amplifiers With Multiple Counter-Pumps," Journal of Ligthwave Technology, vol. 27, no. 4, pp. 944 - 951, April 2009.

[20] B. Bakhshi, M. Vaa, E. A. Golovchenko, H. Li, and G. T. Harvey, "Impact of Gain-Flattening-filter Ripple in LongHaul WDM Systems," in Proceedings of $27^{\text {th }}$ European Conference on Optical Communication (ECOC), Amsterdam, September 2001. 